Supporting Information

\title{
3D Printed Quantum-Dot Nanopixels
}

Jongcheon Bae, Sanghyeon Lee, Jinhyuck Ahn, Jung Hyun Kim, Muhammad Wajahat, Won Suk Chang, Seog-Young Yoon, Ji Tae Kim, Seung Kwon Seol, and Jaeyeon Pyo*

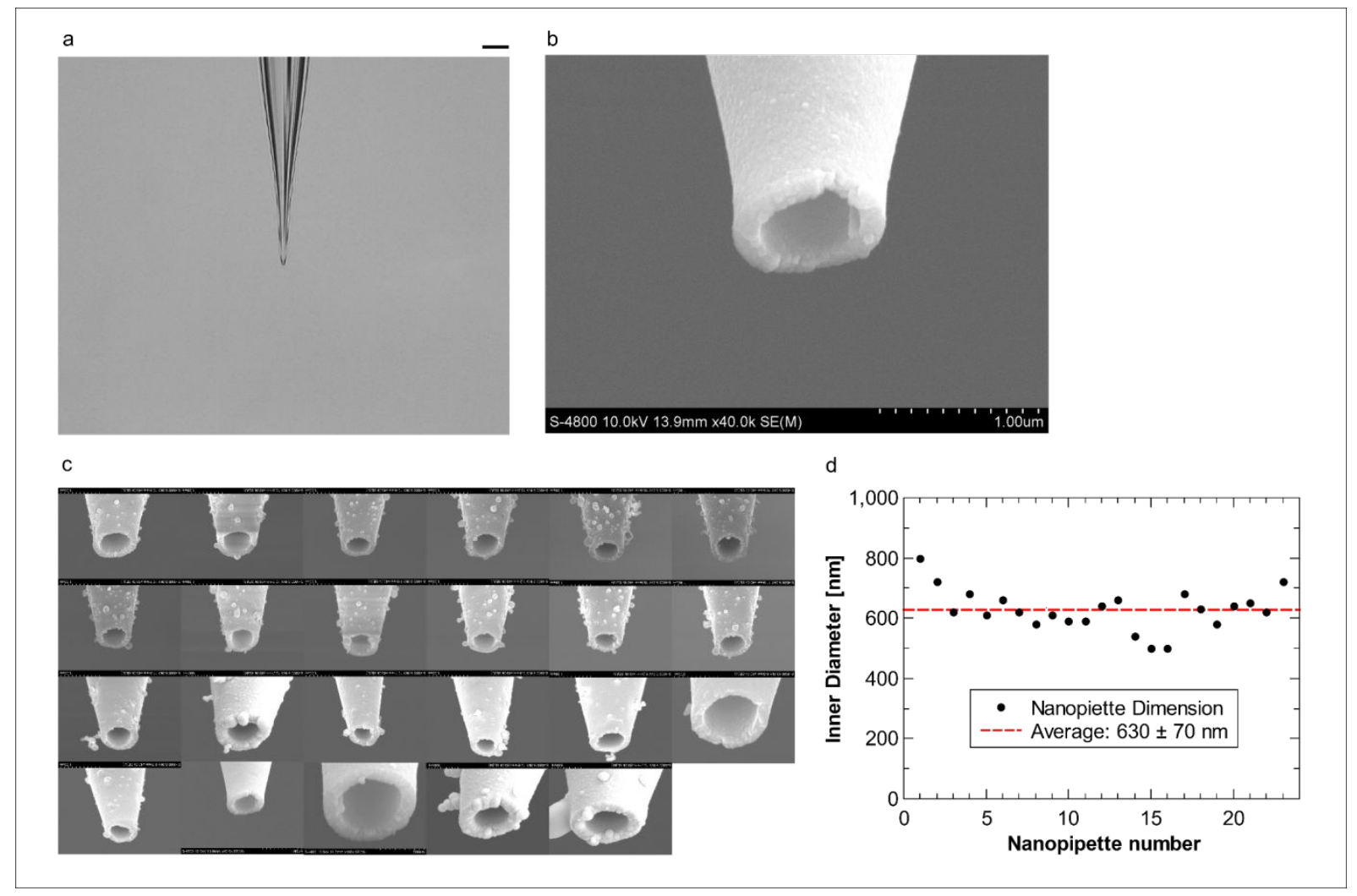

Figure S1. Nanopipette used for 3D printing of the nanopixel. (a) A typical nanopipette observed during the printing process. Scale bar, $10 \mu \mathrm{m}$. (b) Scanning electron microscopy (SEM) image of the nanopipette at a higher magnification. (c) SEM images of a series of nanopipettes for measuring the dimensions. (d) Statistical distribution of the inner diameters of the nanopipettes. 


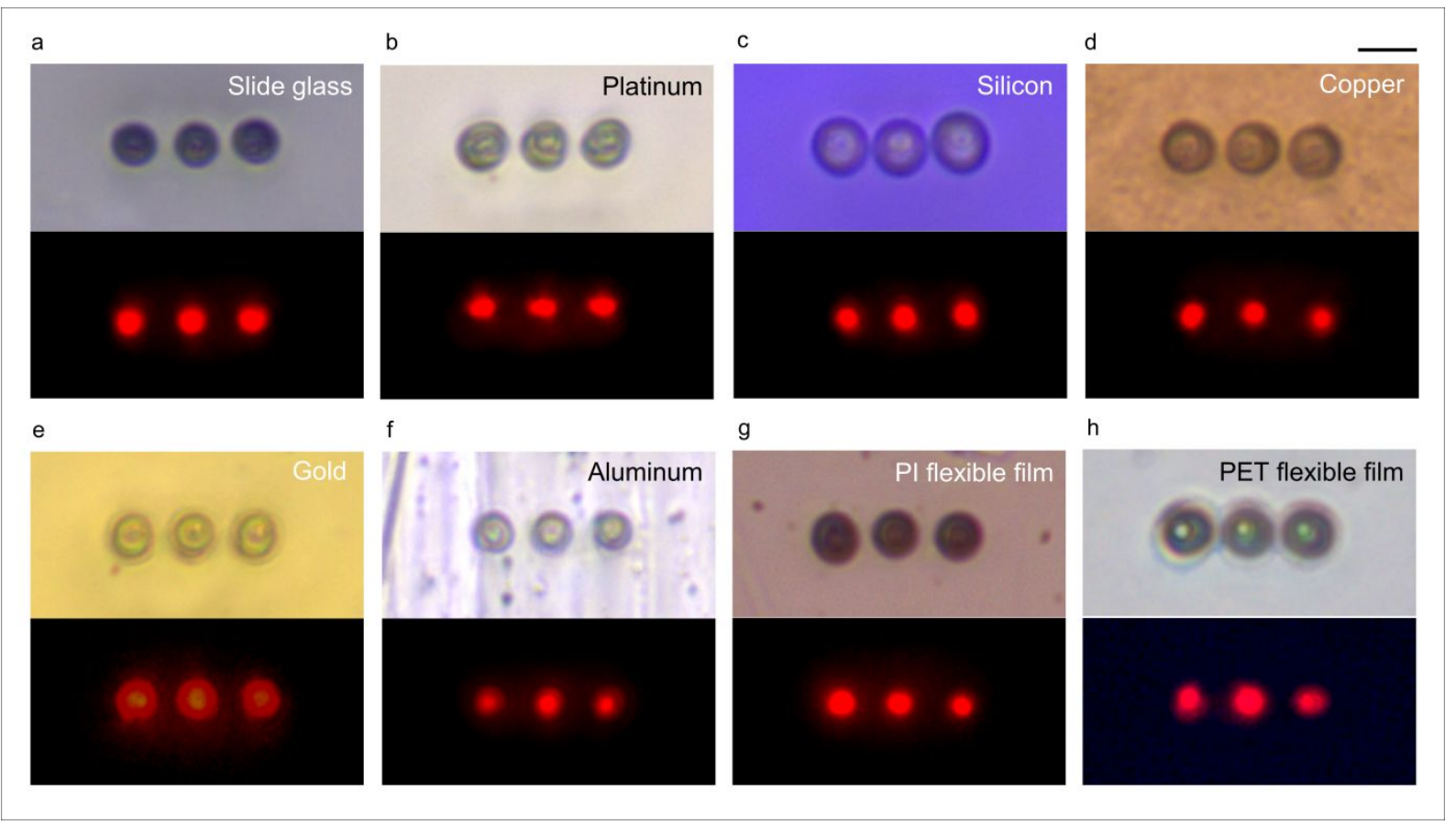

Figure S2. Substrate compatibility. Optical bright-field (BF) and photoluminescence (PL) images of nanopixels printed on (a) slide glass, (b) platinum, (c) silicon, (d) copper, (e) gold, (f) aluminum, (g) polyimide (PI) flexible film, and (h) polyethylene terephthalate (PET) flexible film. Scale bar, $3 \mu \mathrm{m}$ 


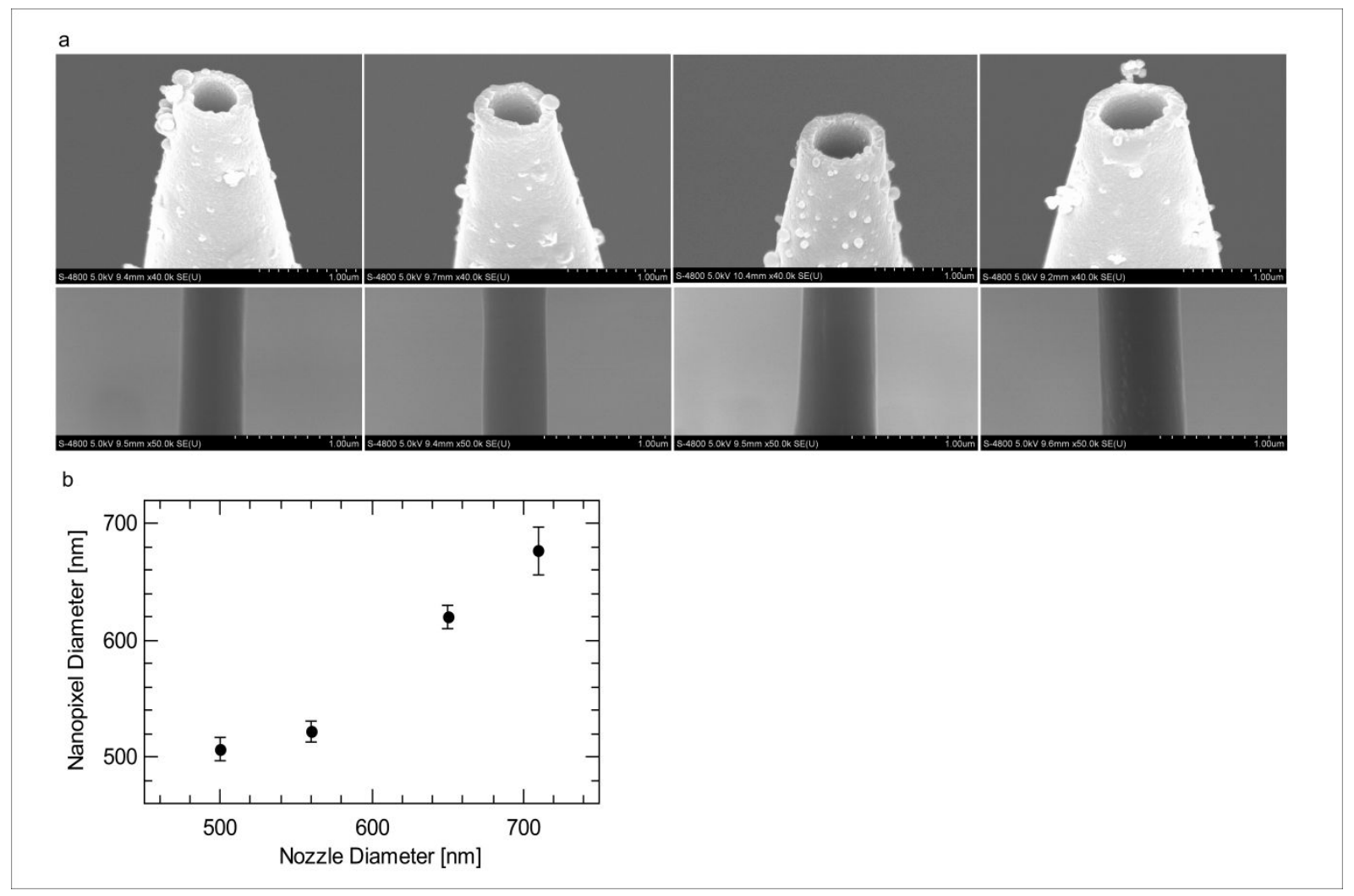

Figure S3. Relation between the diameter of the nanopipette and the nanopixel. (a) SEM images of nanopipettes and corresponding representative nanopixels. (b) Plot of the printed diameter versus the nozzle diameter. 


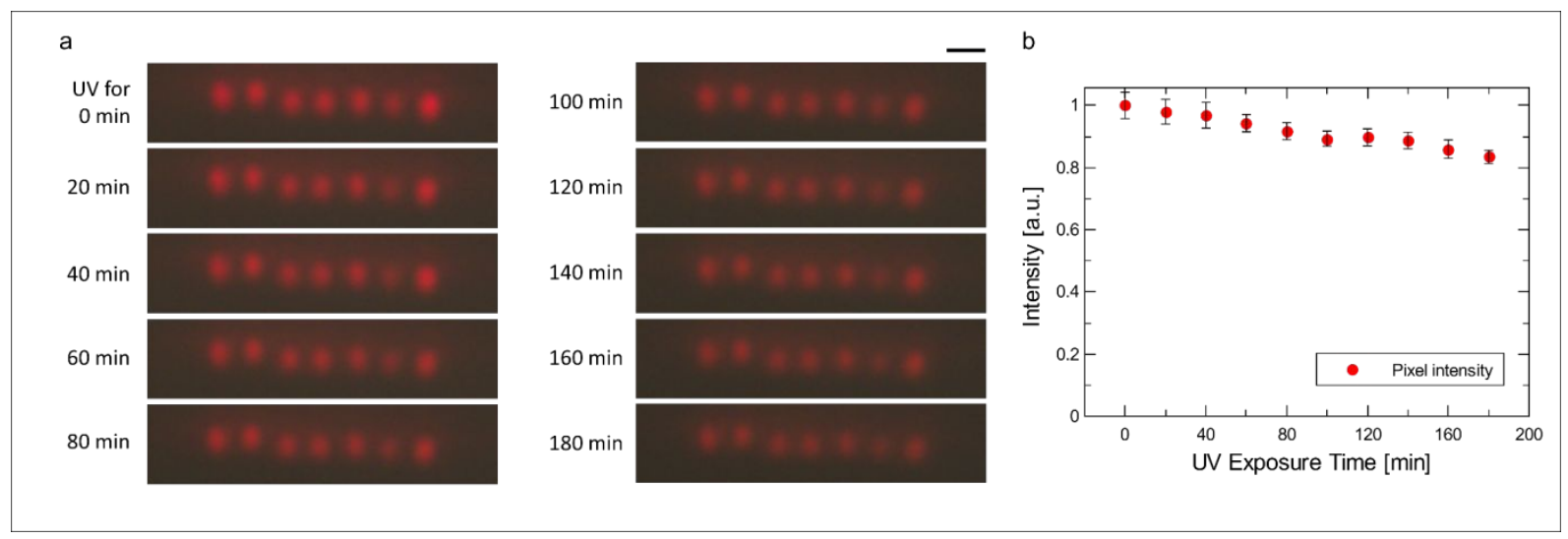

Figure S4. Emission stability. (a) PL images of the printed nanopixels under different UV

exposure times. Scale bar, $3 \mu \mathrm{m}$. (b) Relation between the emission intensity and UV exposure time. 


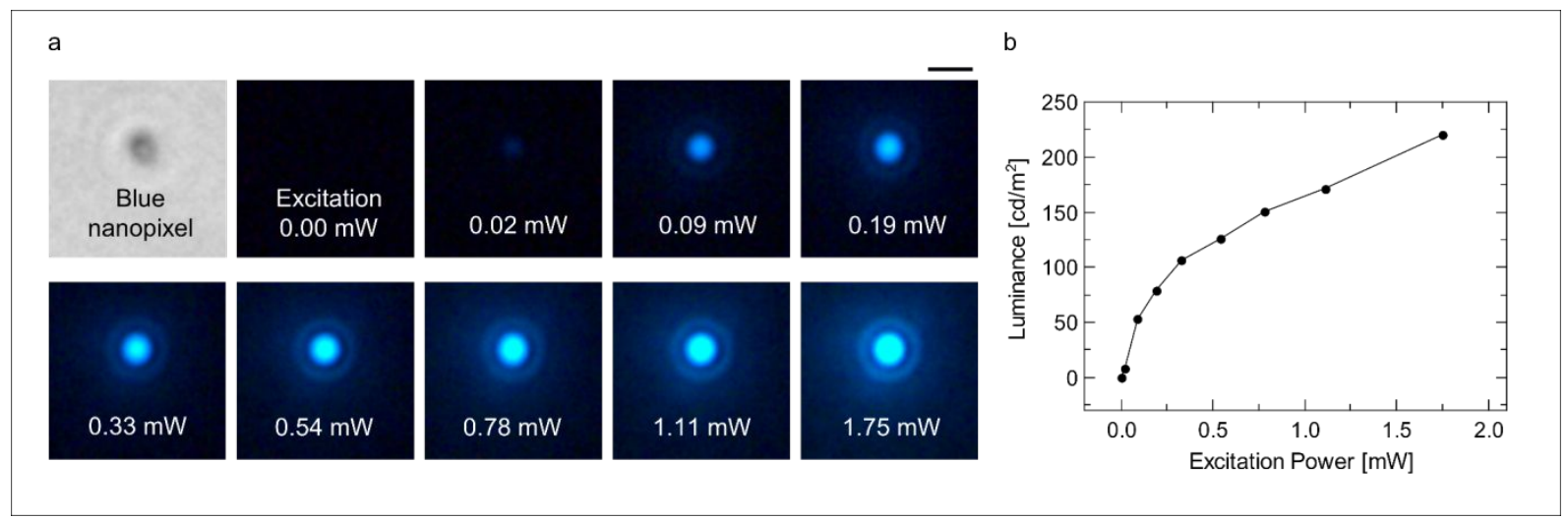

Figure S5. Luminance of the nanopixel. (a) BF and PL images of a blue nanopixel under increasing excitation power. Scale bar, $3 \mu \mathrm{m}$. (b) Relation between the measured luminance of the nanopixel and excitation power. 


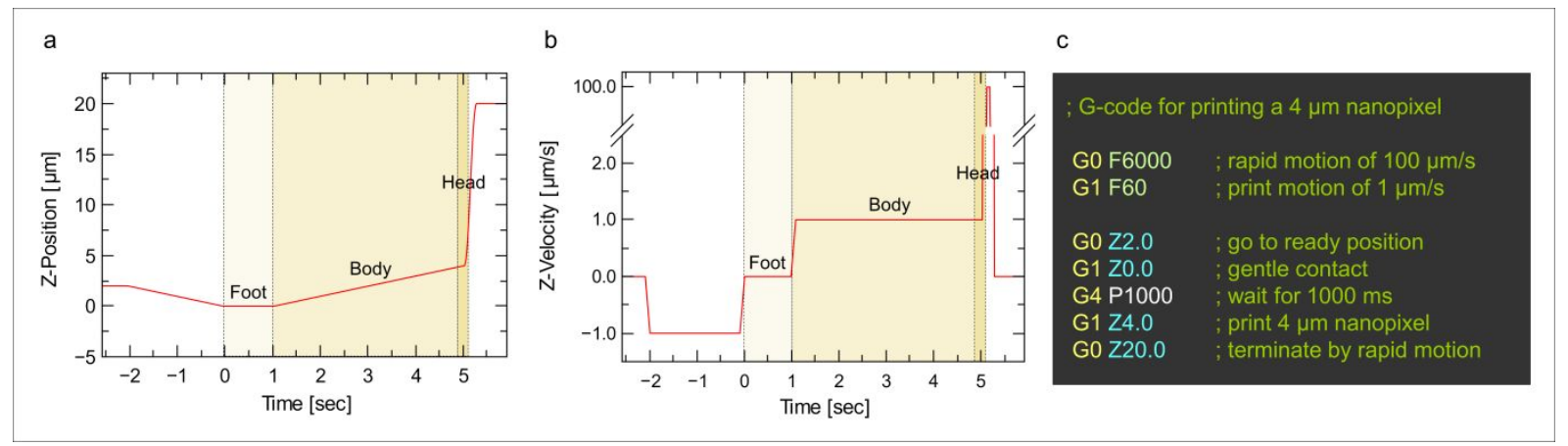

Figure S6. Motion profile for printing the nanopixel. (a) $Z$-axis (height) position, and (b) velocity profiles for printing a $4 \mu \mathrm{m}$ nanopixel. (c) Corresponding g-code with comments. 


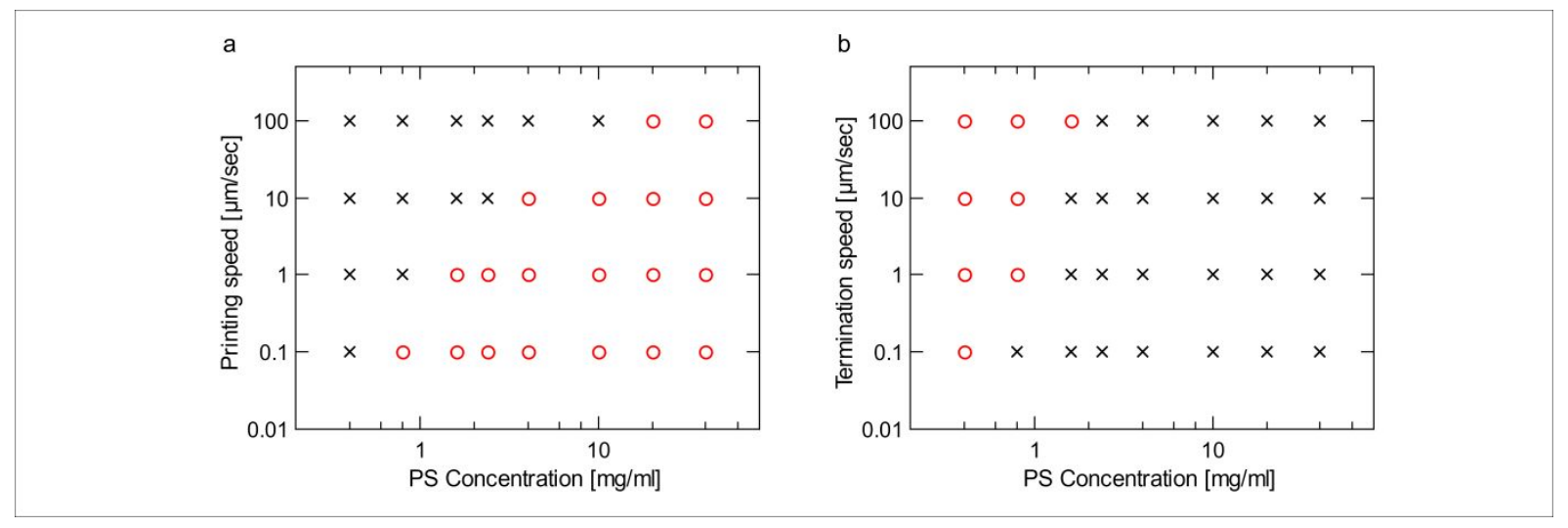

Figure S7. Fabrication chart. Fabrication of the 3D nanopixels requires adequate speed control for both uniform printing and timely termination. (a) Availability of printing at given printing speeds for inks with different compositions. (b) Availability of termination at given termination speeds for inks with different compositions. Circles ( $)$ denote availability, while diagonal crosses $(\times)$ denote unavailability. 


\section{Additional notes}

Motion profile for 3D printing of nanopixels. Each nanopixel was vertically printed at a constant speed of $1 \mu \mathrm{m} / \mathrm{s}$. The printing process was terminated by the rapid removal of the nanopipette at a rate of $100 \mu \mathrm{m} / \mathrm{s}$. To avoid unintentional structural differences, all nanopixels were automatically printed based on the execution of a list of predefined motions in sequence. Every motion in this study used a trapezoidal profile to maximize the distance traveled at a constant speed.

Nozzle control. The temperature of the nanopipette nozzle was not specifically controlled, but it is reasonable to assume it followed the measured room conditions $\left(23^{\circ} \mathrm{C}, 30 \% \mathrm{RH}\right)$. The nanopipette nozzle was position-controlled using precision motorized stages assisted by machine vision.

Simulations. Numerical analyses were performed with the 3D-FDTD method using the opensource software MIT Electromagnetic Equation Propagation (MEEP) (MIT, Boston, MA, USA). The simulation geometry was defined in 3D space based on the experimental observations and according to the simplifications described next. The nanopixel was modeled as a cylinder with a diameter of $600 \mathrm{~nm}$ and refractive index equal to that of polystyrene (1.587). One end of the cylinder was placed in contact with an infinite block (to form the substrate) and had a refractive index equal to that of quartz (1.458). The computational cell was surrounded by the perfectly matched layers to truncate electromagnetic waves. Continuous-wave light at a wavelength of $650 \mathrm{~nm}$ was propagated from the inner parts of the 
nanopixel toward the quartz substrate to simulate light emission at the end-face of the nanopixel. The simulation was run at a spatial resolution of $50 \mathrm{~nm}$ until the field reached steady state. The spatial distribution of the light emission was visualized by plotting the timeaveraged energy density of the electric field on a logarithmic scale. 